\title{
Role of awake prone positioning in patients with moderate-to-severe COVID-19: an experience from a developing country
}

\author{
Iffat Khanum, Fatima Samar, Yousuf Fatimah, Awan Safia, Aziz Adil, Habib Kiren, Nasir Nosheen, \\ Mahmood Faisal, Jamil Bushra \\ Department of Medicine, Section of Infectious Diseases, The Aga Khan University Hospital, Karachi, Pakistan
}

\begin{abstract}
There is limited evidence on the efficacy of awake prone positioning (PP) in non-ventilated patients with COVID-19 who have hypoxemia. We, therefore, aim to describe our experience with the use of early proning in awake, non-intubated patients with confirmed COVID-19. In our retrospective observational study, 23
\end{abstract}

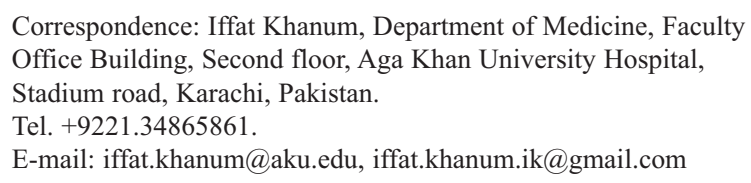

Contributions: All the authors made a substantive intellectual contribution. All the authors have read and approved the final version of the manuscript and agreed to be accountable for all aspects of the work.

Conflict of interest: The authors declare that they have no competing interests, and all authors confirm accuracy.

Conference presentation: European Congress of Clinical Microbiology and infectious Diseases Conference on Coronavirus Disease (ECCVID) Sept 2020, virtual conference.

Ethics approval: The study is approved by ERC of AKUH (ERC number 2020-4764-10530)

Informed consent: As the study was a retrospective review of charts only with no intervention done, all information was available in patient's records and hence consent was not needed.

Availability of data and materials: Data will be available on request by the corresponding author (after taking approval for sharing of data by our ERC).

Received for publication: 11 August 2020.

Accepted for publication: 1 December 2020.

${ }^{\circ}$ Copyright: the Author(s), 2021

Licensee PAGEPress, Italy

Monaldi Archives for Chest Disease 2021; 91:1561

doi: 10.4081/monaldi.2021.1561

This article is distributed under the terms of the Creative Commons Attribution Noncommercial License (by-nc 4.0) which permits any noncommercial use, distribution, and reproduction in any medium, provided the original author(s) and source are credited. patients with confirmed positive PCR test results for Severe Acute respiratory Syndrome Coronavirus-2 (SARS-CoV-2) and hypoxemia that required oxygen therapy with or without non-invasive ventilation were treated with PP. Patients were classified into mild, moderate and severe COVID-19 disease. There were no targeted number of hours for proning per day and patients were kept in prone position according to their tolerance. The primary outcome measure was the avoidance of intubation and secondary outcomes were in-hospital mortality, length of hospital stays and complications related to PP. The mean (standard deviation) age of our cohort was 54.5 (11.7) years, and the majority were males $(21 / 23,91.3 \%)$. Sixty-one per cent $(14 / 23)$ of the patients were suffering from severe disease and $82.6 \%(19 / 23)$ had bilateral lung involvement with interstitial infiltrates. Majority of the patients were prone positioned for a median of 6 days (IQR $4-8$ ). Only one patient required transfer to ICU for mechanical ventilation and subsequently died due to severe ARDS. All 22 patients showed progressive improvement in oxygen requirement and $\mathrm{PF}$ ratio, mostly after 3-5 days of proning. The mean length of hospital stay was 12 days. All patients, except one, were discharged in stable conditions, on room air or on a minimal oxygen requirement of 1-2 liters. No major complication of PP was recorded. Awake prone positioning is a valuable and safe therapeutic adjunct that can be applied in patients with moderate-to-severe COVID19. It can also be included in the home-based management protocols of COVID-19 to improve patient outcomes and mitigate the burden on health care facilities.

\section{Introduction}

The Coronavirus Disease 2019 (COVID-19) crisis, a global pandemic caused by Severe Acute Respiratory Syndrome Coronavirus-2 (SARS-CoV-2) continues to spiral at an alarming rate since its initial detection in Wuhan, China in December 2019. Hypoxemic respiratory failure and acute respiratory distress syndrome (ARDS), leading to mechanical ventilation are common manifestations of this disease process and are associated with a higher morbidity and mortality rate [1]. The fragile and underresourced health care systems of developing countries are facing severe limitations in managing patients during the current pandemic, especially given their constrained capacity and resource shortages [2]. While there is an urgent need of non-invasive and invasive ventilators in low-to-middle-income countries (LMIC), there is also a dire need for cost-effective, scalable modalities that can help in managing acute hypoxemia which, if left untreated, can rapidly progress to ARDS and respiratory failure $[3,4]$.

Prone position ventilation or proning, first proposed in 1974, 
is an authenticated technique used in the treatment of patients with ARDS and has been shown to significantly improve hypoxemia $[5,6]$. Patients with ARDS requiring invasive ventilation are placed in a prone position with their face down and specific prone ventilation protocols have been established to safely perform this manoeuvre $[7,8]$. Prone positioning (PP) improves gas exchange via several mechanisms that improve the ventilation perfusion ratio. It involves the more equal distribution of ventilation with the redistribution of perfusion, recruitment of dorsal alveoli and increased lung volume [9-11]. It may also promote enhanced clearance of secretions from the lungs which improves the ventilationperfusion ratio [10]. The other possible mechanism would be an increased end-expiratory transpulmonary pressure and a more uniform distribution of pleural pressure [12,13].

Earlier studies have established that oxygenation is considerably better with prone position ventilation as compared to the supine position, but data related to its effect on mortality has not been very convincing [14-16]. The prone positioning in severe acute respiratory distress syndrome (PROSEVA) trial demonstrated a significant reduction of 28 days and 90 days of mortality in patients with severe ARDS who received PP [17]. The results of this trial were later supported by succeeding studies including meta-analysis and therefore, PP is now considered as an effective adjunctive therapeutic strategy in ARDS. It decreases mortality especially in patients with severely impaired oxygenation, when initiated early and for a long duration [11,18-21]. Prone positioning is also considered to be effective in COVID-19 related ARDS and has been recommended by surviving sepsis campaign COVID19 management guidelines for critically ill adults [22-24].

The efficacy of PP in awake, spontaneously breathing, nonintubated patients is also documented in literature, but data is limited. Awake prone position in patients with acute hypoxemia has demonstrated beneficial effects including avoidance of intubation [25-27]. A retrospective study by Scaravilli et al. showed that awake proning of non-intubated patients with hypoxemia resulted in a marked improvement in oxygenation and was not associated with serious complications [28].

The role of PP in awake, non-intubated patients with COVID19 is also an area inadequately explored. In the current crisis, where the number of patients with COVID-19 are increasing exponentially with each passing day and health care supplies are running low, awake proning might be a promising, inexpensive and feasible treatment option for patients with moderate to severe ARDS in LMIC.

In this study, we sought to describe our experience with the use of early proning in awake, non-intubated patients with confirmed COVID-19.

\section{Materials and Methods}

This single center, retrospective observational study was conducted at Aga Khan University Hospital (AKUH), Karachi, Pakistan between $1^{\text {st }}$ and $31^{\text {st }}$ May 2020. Established in 1985 , AKUH is one of the largest tertiary care university hospitals of Pakistan ( 740 bedded). It provides a broad range of secondary and tertiary care (both adults and pediatric patients) and caters to a variety of patients referred from all over Pakistan. It also provides both undergraduate and postgraduate training in multiple subspecialties. The nurses-to-patient ratio is 1:6 for ward-beds and 1:4 for high dependency unit overall in the hospital. The internal medicine department admission census is usually between 100 and 170 patients per month. As it is a training institute, there are 71 post graduate trainees to take care of the patients under the supervision of faculty. The physician to patient ratio is between 1:4. There are three respiratory therapists in medicine department who are assigned to take care of patients in high dependency unit. During the COVID-19 pandemic, a part of the hospital has been converted into a designated COVID-19 treatment facility to provide care to critical and non-critical patients. Our hospital has gradually increased its bed capacity in the COVID-19 unit over the period of 4-5 months (beginning from March 2020) where more special care and ICU beds were added after necessary modification like construction of negative pressure rooms.

In our study, we included all patients (18 years or above) who were admitted with moderate to severe COVID-19 and received PP early on after admission as part of their treatment strategy. Patients who received PP with invasive mechanical ventilation were excluded. Moreover, patients with mild COVID-19 or with incomplete data or those who received proning for 24 hours or less were also excluded from our study.

COVID-19 was diagnosed by a nasopharyngeal or oropharyngeal swab for SARS-CoV-2 using real-time reverse transcriptionpolymerase chain reaction (RT-PCR). The severity of disease was graded according to the National guidelines of management of COVID-19 established by the Ministry of Health, Pakistan. According to the guidelines, patients were classified to have mild disease when they had a normal chest X-ray (CXR), stable hemodynamic parameters and maintained oxygen saturation of $\geq 94 \%$ on room air. On the other hand, patients with hypoxemia (oxygen saturation $<94 \%$ but $>90 \%$ ) and chest CXR with infiltrates involving $<50 \%$ of lung fields were classified into the moderate disease category. Patients were said to have severe disease if their oxygen saturation was $<90 \%$ on room air and CXR showed infiltrates involving $>50 \%$ of lung fields.

A team of physicians, comprising of an internist, an infectious diseases specialist and an intensivist took care of patients with COVID-19. The protocol for awake proning was adopted from previously published studies on awake proning [25]. The decision to initiate proning was based on the primary physician's discretion. Patients were placed in prone position with the help of trained nursing staff and a respiratory therapist. There was no pre-fixed targeted number of hours for proning. The frequency of PP and duration of each session of proning was based on patient's comfort level and physician's discretion. The patients were monitored by bedside nurses for their comfort and tolerance of PP. Patients were also counseled about the technique, benefits and complications of awake proning. Along with demographic data, clinical features and CXR findings, sequential organ failure assessment (SOFA) score and $\mathrm{PaO}_{2} / \mathrm{FiO}_{2}(\mathrm{PF})$ ratio were recorded before the initiation of the prone position. Daily $\mathrm{FiO}_{2}$, PF ratio and the number of hours of proning were recorded for each patient. The primary outcome was the avoidance of intubation and secondary outcomes were in-hospital mortality and the length of hospital stay. The study was approved by the Ethical Review Committee (ERC) of the AKUH.

\section{Statistical analysis}

Descriptive analysis was performed; results were reported as numbers with percentages for categorical variables. Continuous variables with normal and non-normal distributions were reported as mean [standard deviation (SD)] and median [interquartile range (IQR)], respectively. Comparison of baseline and discharge was 
done using paired sample t-test. Median values of PF ratio among NIV vs non-NIV group were compared by Mann-Whitney U test. All P-value were two sided and $<0.05$ was considered as statistically significant. SPSS 19.0 (SPSS Inc., Chicago, IL, USA) was used for data analysis.

\section{Results}

A total of 200 patients were admitted with the diagnosis of COVID-19 during May 2020. Twenty patients (10\%) were admitted in ICU and required mechanical ventilation, $120(60 \%)$ in general wards and $60(30 \%)$ in special care unit. Out of 60 patients admitted in special care unit, 23 met the eligibility criteria and were included in our study. The mean (sd) age was 54.5 (11.7) years ranging from 31 to 78 years. The majority of our patients were males $(21 / 23$, $91.3 \%$ ) and the most common comorbid conditions were hypertension and diabetes mellitus. Most of the patients were admitted in the special care unit directly from the emergency department and 14 patients $(60.9 \%)$ required non-invasive ventilation (NIV) at the time of admission. The most common presenting symptoms were fever $(21 / 23,91.3 \%)$, cough $(19 / 23,82.6 \%)$ followed by shortness of breath $(16 / 23,69.6 \%)$ and myalgia $(7 / 23,30.4 \%)$. The median number of days from onset of symptoms to hospitalization was 5 (4-10) days. Moreover, in our cohort, the majority of patients $(60.9 \%$, $14 / 23)$ were suffering from severe disease and $82.6 \%(19 / 23)$ had bilateral lungs involvement with interstitial infiltrates $(21 / 23$, 91.3\%). All patients received COVID-19 related standard medical treatment according to National guidelines with careful evaluation of contraindications to any particular drug (Table 1).

The median SOFA score on the first day before the start of proning was 3 (3-4) while the median duration between admission and initiation of PP was 1 (1-2) day. Most patients were prone positioned for a median of 6 (4-8) days. The minimum number of hours of awake PP tolerated by any patient was 2.5 and maximum number of hours was 16 per day. Only one patient required transfer to ICU for mechanical ventilation and subsequently died due to severe ARDS. The rest of the 22 patients showed progressive improvement in oxygen requirement and PF ratio but responses were variable owing to a difference in the severity of illness amongst patients at the outset. The majority of patients showed improvement in PF ratio after 3-5 days of proning and were successfully tapered off from NIV. The improvement in PF ratio before and at the end of prone positioning was observed in patients with both moderate $(\mathrm{P}=0.008)$ and severe disease $(\mathrm{P}<0.001)$ (Table 2).

In subgroup analysis, patients who were treated with a combination of NIV, oxygen therapy and PP were compared with patients who were treated with PP and oxygen alone, without the use of NIV. We found no evidence of statistically significant improvement in PF ratio between the two groups, except on day 1 of PP $(\mathrm{P}=0.03)$ (Table 3).

All patients tolerated the PP well except for some mild discomfort experienced by most patients. No complication related to PP like facial edema, vomiting, aspiration, intolerance to prone manoeuvre, skin lesion, displacement of venous access, hypotension or arrhythmia was observed in any patient.

The median length of hospital stay was 10 (5-35) days while the median length of special care stay was of 6 (4-8) days. After shifting out of special care units, patients spent a median of 4 (36) days in the ward before being discharged home. All patients except one were discharged in stable condition, on room air or on a minimal oxygen requirement of 1-2 liters.

\section{Discussion}

The mortality with COVID-19 is very high in certain parts of the world, and most patients suffer from hypoxic respiratory failure and ARDS leading to requirement of non-invasive as well as invasive ventilation [29-31].

$\mathrm{PP}$ is being recognized as an effective adjunctive therapy with invasive ventilation in patients with COVID-19 with severe ARDS [32-34]. However, the role of awake PP is not very well defined in non-ventilated patients with COVID-19. Pre COVID-19, the evidence on the use of PP in awake, non-intubated hypoxic patients was limited yet encouraging. Although published in the form of small case series with diverse protocol and different patient population, it has revealed promising results as evidenced by improved

Table 1. Baseline characteristics of study population $(n=23)$.

\begin{tabular}{|c|c|}
\hline & n $(\%)$ \\
\hline Age, years & 54.5 (11.7) \\
\hline $\begin{array}{l}\text { Sex } \\
\text { Male } \\
\text { Female } \\
\end{array}$ & $\begin{array}{c}21(91.3) \\
2(8.7) \\
\end{array}$ \\
\hline $\begin{array}{l}\text { BMI; mean (SD) } \\
\text { Medical conditions } \\
\text { Diabetes mellitus } \\
\text { Hypertension } \\
\text { Ischemic heart disease } \\
\text { Chronic kidney disease } \\
\text { Chronic obstructive pulmonary disease } \\
\text { Malignancy } \\
\text { Immunocompromised state }\end{array}$ & $\begin{array}{c}6(26.1) \\
10(43.5) \\
1(4.3) \\
1(4.3) \\
1(4.3) \\
1(4.3) \\
1(4.3)\end{array}$ \\
\hline Smoker & $2(8.7)$ \\
\hline $\begin{array}{l}\text { Symptoms } \\
\text { Fever } \\
\text { Cough } \\
\text { Myalgia } \\
\text { Runny nose } \\
\text { Sore throat } \\
\text { Dyspnea } \\
\text { Nausea } \\
\text { Vomiting } \\
\text { Abdominal pain } \\
\text { Diarrhea } \\
\text { Headache } \\
\text { Chest tightness } \\
\text { Chills }\end{array}$ & $\begin{array}{c}21(91.3) \\
19(82.6) \\
7(30.4) \\
1(4.3) \\
5(21.7) \\
16(69.6) \\
2(8.7) \\
1(4.3) \\
1(4.3) \\
1(4.3) \\
2(8.7) \\
1(4.3) \\
6(26.1)\end{array}$ \\
\hline $\begin{array}{l}\text { Severity of disease } \\
\text { Moderate } \\
\text { Severe }\end{array}$ & $\begin{array}{c}9(39.1) \\
14(60.9)\end{array}$ \\
\hline $\begin{array}{l}\text { Chest X-ray finding } \\
\text { Unilateral infiltrates } \\
\text { Bilateral infiltrates } \\
\text { Consolidation } \\
\text { Interstitial infiltrates }\end{array}$ & $\begin{array}{c}4(17.4) \\
19(82.6) \\
5(21.7) \\
21(91.3)\end{array}$ \\
\hline $\begin{array}{l}\text { Medication } \\
\text { Systemic steroids } \\
\text { Hydroxycholoroquine } \\
\text { Azithromycin } \\
\text { Tocilizumab } \\
\text { Plasma } \\
\text { Diuretic }\end{array}$ & $\begin{array}{c}21(91.3) \\
16(69.6) \\
9(39.1) \\
13(56.5) \\
2(8.7) \\
22(95.7)\end{array}$ \\
\hline
\end{tabular}


Table 2. Comparison of $\mathrm{PaO}_{2} / \mathrm{FiO}_{2}$ (PF) ratio before and at the end of prone position.

\begin{tabular}{lcccc} 
& PF ratio at baseline & PF ratio at discharge & Mean difference $(95 \% \mathrm{CI})$ & P \\
Moderate disease & $188.7(59.7)$ & $313.1(79.3)$ & $-124.3(-164.3--84.4)$ & $<0.001$ \\
\hline Severe disease & $212.2(39)$ & $306.1(55.8)$ & $-94.11(-155.3--32.85)$ & 0.008 \\
\hline
\end{tabular}

Data are reported as mean (SD).

Table 3. Comparison of median of $\mathrm{PaO}_{2} / \mathrm{FiO}_{2}$ (PF) ratio among NIV versus non-NIV group.

\begin{tabular}{lccc} 
& NIV group & No-NIV group & P-value \\
PF ratio day 1 & $149.5(120-190.5)$ & $229(195-259)$ & 0.003 \\
PF ratio day 3 & $173.5(142-200.2)$ & $325(136.7-392)$ & 0.20 \\
\hline PF ratio day 5 & $236.5(159.7-311.2)$ & $285(133--)$ & 0.84 \\
PF ratio on discharge & $321(252.7-392)$ & $290(270.5-348)$ & 0.47 \\
\hline
\end{tabular}

NIV, non-invasive ventilation; data are reported as mean (SD).

hypoxemia, well tolerability by patients and less or no complications [35]. The proposed mechanisms of improvement in oxygenation by prone position are multifactorial. PP has been witnessed to facilitate the recruitment of alveoli in dorsal lung regions, increase tidal and end-expiratory lung volumes, decrease alveolar shunting as well as improve ventilation-perfusion $(\mathrm{V} / \mathrm{Q})$ ratio via a more homogenous distribution of ventilation $[17,18,36]$.

Awake proning was initially included in the management of patients with COVID-19 in China, the country where the pandemic started. A study from China reported that the employment of this technique resulted in improved mortality and prevented intubation in most patients [37]. This was followed by several small case series reported from other parts of the world which showed the positive effect of early awake proning in management of patients with COVID-19 in terms of clinical improvement and avoidance of intubation $[38,39]$. Few studies also reported that patients only experienced a transient improvement in oxygenation as oxygen saturation levels returned to the baseline after supination [40,41]. None of the patient in moderate COVID-19 disease group in our study developed severe disease. PP might have contributed to improving oxygen requirement of patients with COVID-19; however, this needs further validation in future research.

No adverse events occurred in our cohort and PP emerged as a safe treatment strategy. Previous works also support the fact that it is not associated with an increased incidence of complications in most of the cases $[41,42]$. In the current crisis, many patients with COVID-19 are being managed at home with oxygen therapy due to limited capacity of health care facilities, especially in resourcepoor countries. Our results support the fact that patients and their families can easily be educated to initiate early awake proning at home along with close monitoring of oxygen saturation. It may prove to be an excellent adjunctive therapeutic strategy that requires minimal resources and can be included in the management protocol of home-based therapy of patients with COVID-19 in early hypoxemic stage of illness. However, our study has several limitations. This is a retrospective single centre study with a small sample size and no control group. Moreover, we could not elicit the minimum duration of prone position required to attain a significant clinical improvement as there were no pre-fixed targeted hours per day for patients to remain in the prone position. Further multicentre studies with a larger sample size and pre-defined awake proning protocol as well as control groups, preferably ran- domized control trials are required to validate and generalize our results or draw any definitive conclusions.

\section{Conclusions}

As there is no effective therapy available for COVID-19 to date, clinicians are in a constant struggle to improve supportive care. Awake PP might prove to be an effective adjunct therapeutic option that can be applied in patients with moderate to severe COVID-19. It is a safe and cost-effective measure that has attained widespread popularity amongst most physicians due to its ease of application and encouraging results. It can also be initiated at home as part of management of patients with less severe hypoxemia who cannot be admitted in hospitals, thus decreasing the load on health care facilities. However, further studies with larger sample size and control groups are needed to further validate our findings.

\section{References}

1. Kashani KB. Hypoxia in COVID-19: Sign of severity or cause for poor outcomes. Mayo Clin Proc 2020 ;95:1094-6.

2. Siow WT, Liew MF, Shrestha BR et al. Managing COVID-19 in resource-limited settings: critical care considerations. Crit Care 2020;24:167.

3. Guérin C, Lévy P. Easier access to mechanical ventilation worldwide: an urgent need for low income countries, especially in face of the growing COVID-19 crisis. Eur Respir J 2020;55:2001271.

4. Fan E, Beitler JR, Brochard L, et al. COVID-19-associated acute respiratory distress syndrome: is a different approach to management warranted? Lancet Respir Med 2020;8:816-21.

5. Bryan A. Conference on the scientific basis of respiratory therapy. Pulmonary physiotherapy in the pediatric age group. Comments of a devil's advocate. Am Rev Respir Dis 1974;110:143-4.

6. Fernandez R, Trenchs X, Klamburg J, et al. Prone positioning in acute respiratory distress syndrome: a multicenter randomized clinical trial. Intensive Care Med 2008;34:1487-91. 
7. Oliveira VM, Weschenfelder ME, Deponti GN, et al. Good practices for prone positioning at the bedside: Construction of a care protocol. Rev Assoc Med Bras 2016;62:287-93.

8. Oliveira VM, Piekala DM, Deponti GN, et al. Safe prone checklist: construction and implementation of a tool for performing the prone maneuver. Rev Bras Ter Intensiva 2017;29:131-41.

9. Henderson WR, Griesdale DE, Dominelli P, Ronco JJ. Does prone positioning improve oxygenation and reduce mortality in patients with acute respiratory distress syndrome? Can Respir J 2014;21:213-5.

10. Johnson NJ, Luks AM, Glenny RW. Gas exchange in the prone posture. Respir Care 2017;62:1097-110.

11. Scholten EL, Beitler JR, Prisk GK, Malhotra A. Treatment of ARDS with prone positioning. Chest 2017;151:215-24.

12. Kumaresan A, Gerber R, Mueller A, et al. Effects of prone positioning on transpulmonary pressures and end-expiratory volumes in patients without lung disease. Anesthesiology 2018;128:1187-92.

13. Guérin C.Prone ventilation in acute respiratory distress syndrome.Eur Respir Rev 2014;23:249-57.

14. Abroug F, Ouanes-Besbes L, Elatrous S, Brochard L. The effect of prone positioning in acute respiratory distress syndrome or acute lung injury:a meta-analysis. Areas of uncertainty and recommendations for research. Intensive Care Med 2008;34:1002-11.

15. Sud S, Sud M, Friedrich JO, Adhikari NK. Effect of mechanical ventilation in the prone position on clinical outcomes in patients with acute hypoxemic respiratory failure: a systematic review and meta-analysis. CMAJ 2008;178:1153-61.

16. Alsaghir AH, Martin CM. Effect of prone positioning in patients with acute respiratory distress syndrome: a metaanalysis.Crit Care Med 2008;36:603-9.

17. Guérin C, Reignier J, Richard J-C, et al. Prone positioning in severe acute respiratory distress syndrome. N Engl J Med 2013;368:2159-68.

18. Kallet RH. A comprehensive review of prone position in ARDS.Respir Care 2015;60:1660-87.

19. Munshi L, Del Sorbo L, Adhikari NK, et al. Prone position for acute respiratory distress syndrome.A systematic review and meta-analysis. Ann Am Thorac Soc 2017:S280-8.

20. Mora-Arteaga JA, Bernal-Ramírez OJ, Rodríguez SJ. The effects of prone position ventilation in patients with acute respiratory distress syndrome. A systematic review and metaanalysis. Med Intensiva 2015;39:359-72.

21. Dalmedico MM, Salas D, Oliveira AM, et al. Efficacy of prone position in acute respiratory distress syndrome: overview of systematic reviews. Rev Esc Enferm USP 2017;51:e03251.

22. Alhazzani W, Møller M, Arabi Y, et al. Surviving Sepsis campaign: Guidelines on the management of critically ill adults with coronavirus disease. Crit Care Med 2020;48:e440-69.

23. Dondorp AM, Hayat M, Aryal D, et al. Respiratory support in COVID-19 patients, with a focus on resource-limited settings.A m J Trop Med Hyg 2020;102:1191-7.

24. Valter C, Christensen AM, Tollund C, SchØnemann NK. Response to the prone position in spontaneously breathing patients with hypoxemic respiratory failure. Acta Anaesthesiol Scand 2003;47:416-8.
25. Feltracco P, Serra E, Barbieri S, et al. Noninvasive high-frequency percussive ventilation in the prone position after lung transplantation. Transplant Proc 2012;44: 016-21.

26. Feltracco P, Serra E, Barbieri S, et al. Non-invasive ventilation in prone position for refractory hypoxemia after bilateral lung transplantation. Clin Transplant 2009;23:748-50.

27. Scaravilli V, Grasselli G, Castagna L, et al. Prone positioning improves oxygenation in spontaneously breathing nonintubated patients with hypoxemic acute respiratory failure: A retrospective study. J Crit Care 2015;30:1390-4.

28. Suleyman G, Fadel RA, Malette KM, et al. Clinical characteristics and morbidity associated with coronavirus disease 2019 in a series of patients in Metropolitan Detroit. JAMA Netw Open 2020;3:e2012270.

29. Liang WH, Guan WJ, Li CC, et al. Clinical characteristics and outcomes of hospitalised patients with COVID-19 treated in Hubei (epicentre) and outside Hubei (non-epicentre): a nationwide analysis of China. Eur Respir J 2020;55:2000562.

30. Aggarwal A, Shrivastava A, Kumar A, Ali A. Clinical and epidemiological features of SARS-CoV-2 patients in SARI ward of a tertiary care centre in New Delhi. J Assoc Physicians India 2020;68:19-26.

31. Meng L, Qiu H, Wan L, et al. Intubation and ventilation amid the COVID-19 outbreak: Wuhan'experience. Anesthesiology 2020;132:1317-32.

32. Ghelichkhani P, Esmaeili M. Prone position in management of COVID-19 patients; a commentary. Arch Acad Emerg Med 2020;8:e48.

33. Sugimoto R, Kenzaka T, Fujikawa M, et al. Humidifier use and prone positioning in a patient with severe COVID-19 pneumonia and endotracheal tube impaction due to highly viscous sputum. Cureus 2020;12:e8626.

34. Purvis P, Francis O. Prone position ventilation in non-intubated, spontaneously ventilating patients: New guidance from the Intensive Care Society (UK) and existing evidence. J Intensive Care Soc 2020;21:1-2.

35. Pelosi P, Brazzi L, Gattinoni L. Prone position in acute respiratory distress syndrome. Eur Respir J 2002;20:1017-28.

36. Sun Q, Qiu H, Huang M, Yang Y. Lower mortality of COVID19 by early recognition and intervention: experience from Jiangsu province. Ann Intensive Care 2020;10:33.

37. Ng Z, Tay WC,Ho CHB. Awake prone positioning for non-intubated oxygen dependent COVID-19 pneumonia patients. Eur Respir J 2020;56:2001198.

38. Xu Q, Wang T, Qin X, et al.Early awake prone position combined with high-flow nasal oxygen therapy in severe COVID19:a case series. Crit Care 2020;24:250.

39. Elharrar X, Trigui Y, Dols AM, et al. Use of prone positioning in non-intubated patients with COVID-19 and hypoxemic acute respiratory failure. JAMA 2020;323:2336-8.

40. Sartini C, Tresoldi M, Scarpellini P, et al. Respiratory parameters in patients with COVID-19 after using noninvasive ventilation in the prone position outside the intensive care unit. JAMA 2020;323:2338-40.

41. Messerole E, Peine P, Wittkopp S, et al. The pragmatics of prone positioning. Am J Respir Crit Care Med 2002;165: 1359-63. 